\title{
Homing performance of anosmic pigeons
}

\author{
RON L. SNYDER and CARL D. CHENEY \\ Institute of Animal Behavior, Utah State University, Logan, Utah 84322
}

Two homing experiments were conducted with control pigeons and pigeons whose nares were obstructed. Anosmia reduced return success and extended flight times. Familiarity with the route cancelled much of the difference between groups. Olfaction does appear to be a sense mode useful to homing pigeons.

Homing pigeons and migrating birds use, but may not require, optical cues to find their way (Schlichte \& Schmidt-Koenig, 1972). Nonvisual alternatives which have received experimental attention include Coriolis forces (Ising, 1946), low-frequency sound waves (Griffin, 1969), inertial navigational means (Barlow, 1964), and the geomagnetic field (Yeagley, 1951). Only one of these possible nonoptical sources of navigational information, the geomagnetic field, seems to affect the orientation of birds (Wiltschko \& Wiltschko, 1972), and its role remains controversial. Since discrimination within the magnetic field presumes an as yet unknown sensory basis, a more parsimonious nonvisual candidate may be olfaction. For instance, Grubb (1974) found that the ability of Leach's petrels (Oceandrama leucorrhoe) to return to a nesting burrow was disrupted if their olfactory bulbs were surgically ablated or if their external nares were plugged. Papi, Fiore, Fiaschi, and Benvenuti (1973) have also shown that olfactory impairment while at the loft disrupts homing performance of pigeons (Columba livia).

We investigated the role of olfaction in the performance of homing pigeons using the sealed nares method. The procedure was considered to be less behaviorally disruptive than either surgical methods (Benvenuti, Fiaschi, Fiore, \& Papi, 1973) or washing of the olfactory receptor surfaces with acid. Our results suggest that elimination of olfactory information does disrupt both the orientation and homing ability of pigeons, but that the effect is attenuated if the birds have previously been familiarized with the terrain over which they must fly in order to return to the loft.

\section{EXPERIMENT I}

\section{Method}

Subjects and Procedure. In the first experiment 46 mixedbreed pigeons were used. The birds' ages ranged from 6 to 13 months, and they were allowed to fly about the loft from the time they fledged. They initially experienced flights returning

We thank Dr. Balph, Director, Green Canyon Ecology Research Center, Utah State University, for facilities and Debbie Muller and Joe Iste for assistance. Reprints may be obtained from R. Snyder, Department of Design and Exhibitions, Brookfield Zoo, Brookfield, Illinois 60513. to the loft from a release point 9.4 kilometers south of the loft (Point $A$ in Figure 1). After each bird had successfully returned to the loft within $30 \mathrm{~min}$ on five consecutive trials, the pigeons were randomly divided into two groups of 23 birds each, Group 1 and Group 2. Before removal from the loft on each subsequent test release, all birds in one group were olfactorily impaired. The nares were sealed by the insertion of a modeling clay plug, held in place with a short length of plastic tape coated with waterproof cement (Grubb, 1974). The control group for each test received only the tape with a hole positioned over the nares. None of the birds appeared to be distracted by the treatment.

Figure 1 shows the location of the test release points, numbered in order of occurrence (1-5). Each point was approximately 18.0 kilometers from the loft. Group 1 birds were nasally obstructed before the first and third releases, while Group 2 birds were obstructed before the second and fourth. Each homing test trial was preceded by a 3-day period of free feeding and 1 day of food deprivation and restriction to the loft. The birds were individually released in random order following the disappearance of the previously released bird. No pigeons were nasally occluded during the fifth trial in order to assess any homing success differences between groups, independent of the nasal occlusion treatments.



Figure 1. Training and release points. The loft was located 2 kilometers northwest of Logan, Utah at an elevation of $1,373 \mathrm{~m}$. The study area is predominately rural farmland with some developed marshland. 


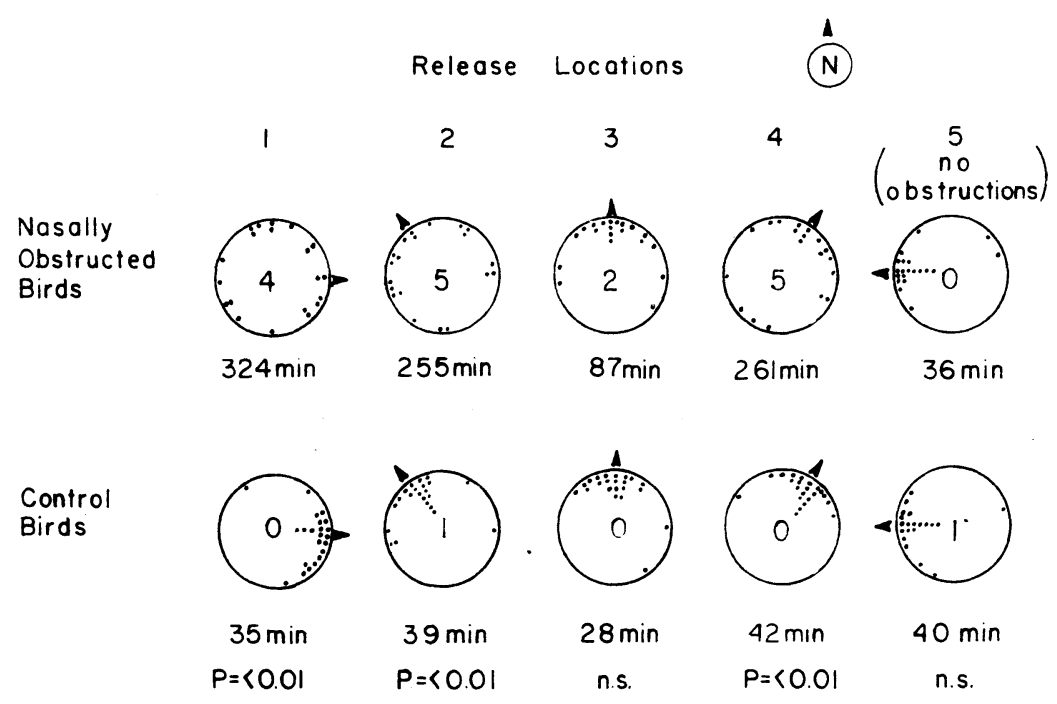

Figure 2. Vanishing bearings for each bird and mean flight time for each homing trial in Experiment I. Each dot represents the direction a pigeon was heading when it disappeared over the horizon. Numbers within the circles represent birds which roosted after release. Release point numbers correspond with Figure 1. The total number of birds for each trial decreases from 46 to 40 due to loss of birds during the experiment. Arrows on the circles point toward the loft.

Results

Figure 2 records the vanishing bearings of all the birds from each release point. The occluded birds usually disappeared at nearly random compass points or roosted near the release point, while the control birds tended to disappear bearing toward the loft. The chisquare test method of Batschelet (1965) was used to assess the significance of these differential bearings. Figure 2 also shows the mean flight time for both groups. Anosmic birds often took 10 times as long to reach the loft as did the untreated birds. Six pigeons with obstructed nares were lost during this experiment, while none of the control birds failed to return. The disappearing bearing and flight time data from the fifth release point, where no birds were anosmic, indicates that the groups were similar in homing ability and that the occlusion experience apparently does not substantially interfere with subsequent normal homing behavior.

The nasal plugs had a marked effect upon the birds' homing performance, but the flight time data revealed that both groups of birds returned to the loft more quickly from the south (Release Point 3 ) than from the other release points. Also, the disappearing bearings were similar for obstructed and nonobstructed birds in this trial. The route back to the loft from this release point required the birds to fly over the same terrain they had flown over during pre-experimental training. Therefore, we conducted a second experiment in order to investigate the possibility that familiarity of the route to the loft might attentuate the disruptive effects of the nasal occlusions on homing.

\section{EXPERIMENT II}

\section{Method}

Subjects and Procedure. First, 40 different mixed-breed birds were trained (by being released at gradually more distant points) to return from a release point 28.0 kilometers southwest of the loft (Point $B$ in Figure 1). The pigeons were then

randomly assigned to four groups of 10 birds each. After the same preflight feeding program as in the first experiment, Groups 1 and 2 were individually released from a point 38.4 kilometers southwest of the loft (Point $C$ in Figure 1). Group 1 subjects were nasally obstructed, while Group 2 birds received only the control treatment. Group 3 (anosmic) and Group 4 (control) were released from a point 39.0 kilometers northeast of the loft (Point D in Figure 1).

\section{Results}

Figure 3 shows the disappearing bearings of all four groups and their mean flight times. As in Experiment I, the nasally occluded birds disappeared at varied compass points, although there was a slight bias toward the south for both occlusion groups. The flight times show that both nonoccluded groups returned to the loft earlier than the occluded birds, with those that were familiar with some of the route returning first. All of the nasally obstructed birds flying over familiar terrain returned. Three of the 10 anosmic/unfamiliar terrain birds were lost.

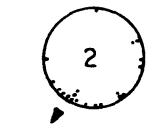

Obstructed

Unfamiliar Route $3470 \mathrm{~min}$

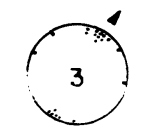

Obstructed Familiar Route $620 \mathrm{~min}$
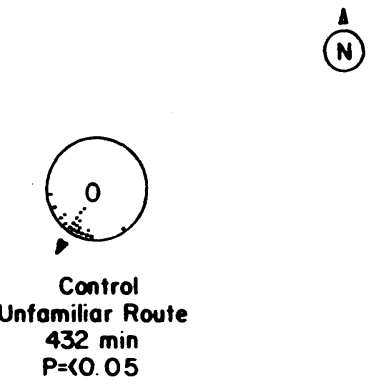

Figure 3. Vanishing bearings and mean flight times for Experiment II. Flight time includes only daylight hours. 


\section{DISCUSSION}

Nasal obstructions force pigeons to breath through their beaks, and this could account for some of the differential homing performance found in the present study. However, the anosmic birds released from Point 3 in Experiment $I$ and the anosmic birds flying over familiar terrain in Experiment II performed better than the anosmic birds flying over unfamiliar terrain. If nares-obstruction discomfort was primarily responsible for poor homing performance, no such difference should have occurred.

We conclude that familiarity with terrain not only improves the homing success of nonoccluded birds but also attenuates the effects of nasal occlusions. Therefore, these results are in accord with findings that olfactory cues may be an important source of navigational information for homing pigeons, but they also indicate that visual cues may be substituted when they are informative. Whether this relationship holds during longdistance homing (pigeons have returned from distances as great as 1,500 kilometers) remains to be shown. It is probably the case that visual cues, such as the "sun compass" (Kramer, 1952), become more important at great distances. These results do not contradict previous studies which have investigated other alternatives to visual cues in homing. The importance of olfaction in bird homing may be complimentary to the data showing disruptive effects of artificial magnetic fields (e.g., Wiltschko \& Wiltschko, 1972; Yeagley, 1951) on bird orientation. Such fields may influence air-borne molecules and thereby modify their olfactory information-bearing capacity or they may have a direct effect on the olfactory system of the birds.

\section{REFERENCES}

BARLOw, J. S. Inertial navigation as a basis for animal navigation. Journal of Theoretical Biology, 1964, 6, 76-117.
Batschelet, E. Statistical methods for the analysis of problems in animal orientation and certain biological rhythms. American Institute of Biological Science, 1965, Monograph 1, 57.

Benvenuti, S., Fiaschi, V., Fiore, L., \& Papi, F. Homing performance of inexperienced and directionally trained pigeons subjected to olfactory nerve section. Journal of Comparative Physiology, 1973, 83, 81-92.

GRIFFIN, D. R. The physiology and geophysics of bird navigation. Quarterly Review of Biology, 1969, 44, 255-276.

GRUB B, T. C., JR. Olfactory navigation to the nesting burrow in Leach's Petrel (Oceanodroma laucorrhoa). Animal Behavior, 1974, 22, 192-202.

IsıNG, G. Die physikalische Möglichkeit eines tierischen Orientierungssinnes auf Basis der Erdrotation. Arkives of Mathematical Astro Fysik, 1946, 32A, 1-23.

Kramer, G. Experiments in bird navigation. Ibis, 1952, 94, 265-285.

Papi, F., Fiore, L., Fiaschi, V., \& Benvenuti, S. An experiment for testing the hypothesis of olfactory navigation in homing pigeons. Journal of Comparative Physiology, 1973, 83, 93-102.

Schlichte, H. J., \& Schmidt-Koenig, K. Homing in pigeons with impaired vision. Proceedings of the National Academy of Science, 1972, 69, 2446-2447.

Wiltschко, W., \& Wiltschко, R. Magnetic compass of European robins. Science, 1972, 176, 62-64.

YeAGLEY, H. L. A preliminary study of a physical basis of bird navigation. II. Journal of Applied Physiology, 1951, 22, 746-760. 